\title{
Expanding the Topological Space of Bioactive Peptides
}

\author{
Jean-Louis Reymond* and Tamis Darbre
}

\begin{abstract}
Naturally occurring peptides and proteins consist almost exclusively of linear or cyclic polypeptide chains. Our group explores the chemical space of peptides by redesigning their topology through the introduction of branching points in the peptide chain. Branched peptides are generally resistant to proteolysis and display remarkable biological properties by multivalency effects (peptide dendrimers) and conformational rigidity (polycyclic peptides). We review our recent progress in peptide dendrimers as enzyme models, biofilm inhibitors, antimicrobials, DNA transfection and cell-penetrating agents, and in the synthesis and characterization of bicyclic peptides as new scaffolds for drug design.
\end{abstract}

Keywords: Bioactive peptides

\section{Introduction}

Naturally occurring peptides and proteins consist almost exclusively of linear or cyclic polypeptide chains translated from genes through the ribosome. Peptide research generally focuses on these peptides by investigating their synthesis as well as alterations in the sequence or the nature of the amino acid building blocks, such as single deletions, insertions and substitutions, or even complete replacement of residues by non-natural, enantiomeric or $\beta$-amino acids, or other types of building blocks or peptide linkages. These modifications are introduced to increase conformational rigidity, biological activity and metabolic stability, and mostly rely on solid-phase peptide synthesis (SPPS), a robust and versatile laboratory method that has the ability to deliver products in the laboratory as well as on an industrial scale. ${ }^{[1]}$ Synthesizing macromolecules from amino acids by SPPS is not only technically straightforward, but also a strategic choice when searching for bioactive molecules given the substantial track record of synthetic peptides as therapeutics. ${ }^{[2]}$

Rather than modifying amino acids in peptide sequences, our group explores the peptide chemical space by redesigning the topology of the peptide chain. Although the importance of topology for biological activity is evident in naturally occurring peptides such as cysteine knot proteins ${ }^{[3]}$ and lantibiotics, ${ }^{[4]}$ relatively little attention has been paid to the systematic synthetic exploration of peptide topologies. ${ }^{[5]}$ In analogy to the structural diversity of covalent bond networks between atoms within organic molecules, ${ }^{[6]}$ one can design topologically diverse peptides from mathematical graphs by assigning amino acids to graph nodes and peptide bonds to graph edges. While most amino acids have a maximum of two peptide linkage points and are therefore only suitable for peptide equivalents of linear or cyclic graphs, using diamino acids (e.g. lysine) or amino diacids (e.g. glutamate) as branching points in a peptide enables the realization of a variety of additional graph topologies. In line with this general principle, we are studying two types of branched peptide scaffolds, namely peptide dendrimers and polycyclic peptides (Fig. 1).[7]

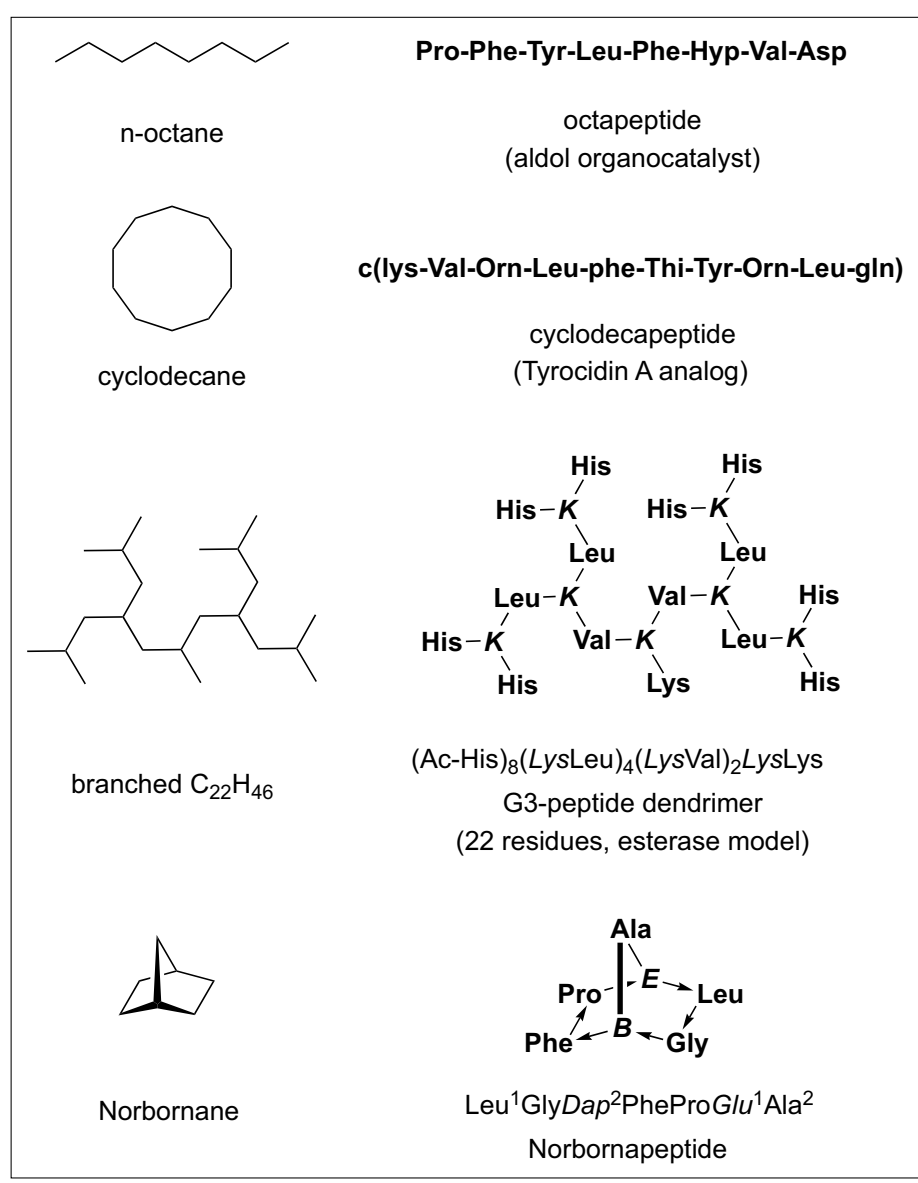

Fig. 1. Topologically diverse alkanes and their peptide analogs: an octapeptide aldolase catalyst, ${ }^{[8]}$ an antimicrobial cyclodecapeptide, ${ }^{[9]}$ a G3 esterase peptide dendrimer, ${ }^{[10]}$ and a norbornapeptide.[11] Branching residues are marked in italics, $B$ indicates the branching 2,3-diaminopropanoic acid (Dap). All C-termini are carboxamide. In the norbornapeptide the arrow indicates a $\mathrm{C} \rightarrow \mathrm{N}$ backbone peptide bond.

${ }^{*}$ Correspondence: Prof. Dr. J.-L. Reymond Department of Chemistry and Biochemistry University of Bern

Freiestrasse 3, CH-3012 Bern

E-mail: jean-louis.reymond@ioc.unibe.ch 


\section{Peptide Dendrimers}

\section{Combinatorial Assays and Enzyme Models}

We initially studied peptide dendrimers as enzyme models ${ }^{[12]}$ extending on previous work with catalytic antibodies[13] and high-throughput screening enzyme assays. ${ }^{[14]}$ The experiments were based on an efficient library design algorithm for peptides, cyclic peptides and peptide dendrimers allowing the derivation of the sequence of the product on a combinatorial synthesis bead from its amino acid composition as given by quantitative amino acid analysis. ${ }^{[8]}$ The protocol was suitable for a variety of 'on-bead' screening assay with colorimetric or fluorogenic reagents such as enzyme substrates or labeling reagents. ${ }^{[15]}$ We also developed an 'off-bead' assay protocol in which the beads were subjected to a controlled UV-irradiation without solvent to partially cleave a photolabile linker. The beads were then spread on a solid support impregnated with an assay reagent, allowing small amounts of peptide to diffuse from the beads to the support. Beads carrying an active sequence were revealed by a reagent halo, e.g. a fluorescent halo on a silica gel plate impregnated with a fluorogenic substrate solution when searching for catalytic activities. ${ }^{[16]}$ Due to limited diffusion into the support the beads still contained sufficient amounts of peptide for sequence determination by amino acid analysis. These combinatorial projects produced various esterase and aldolase peptide and peptide dendrimer enzyme models. ${ }^{[17]}$

Screening combinatorial libraries also allowed us to identify peptide dendrimers binding to cobalamin by coordination to cobalt. ${ }^{[18]}$ In these dendrimers metal coordination by a cysteine thiolate at the dendrimer core was found to be remotely con- trolled by electrostatic charges in the outer dendrimer branches. ${ }^{[19]}$ The same effect controlled metal coordination by bipyridine ligands within polyanionic or polycationic peptide dendrimers. ${ }^{[20]}$ In search for the realization of a metallopeptide dendrimer enzyme model, we recently investigated if the coordination of a bipyridine ligand by $\mathrm{Fe}(\mathrm{II})$ could be similarly tuned by appending polyanionic or polycationic G3-peptide dendrimers using a thioether ligation step, a convergent method particularly well suited for the assembly of protein-sized dendrimers.[21] Indeed the bipyridine peptide dendrimer BP1 formed a 1:1 Fe.BP1 complex at $\mathrm{pH}$ 6.5. This complex acted as a peroxydase enzyme model and catalysed the oxidation of $o$-phenylene diamine with hydrogen peroxide, while the corresponding $\mathrm{Fe}\left(\right.$ bipy) ${ }_{3}$ complex was inactive (Fig. 2a). ${ }^{[22]}$ Interestingly, charge neutralization by mutating all twenty-four glutamate residues present in the dendrim-

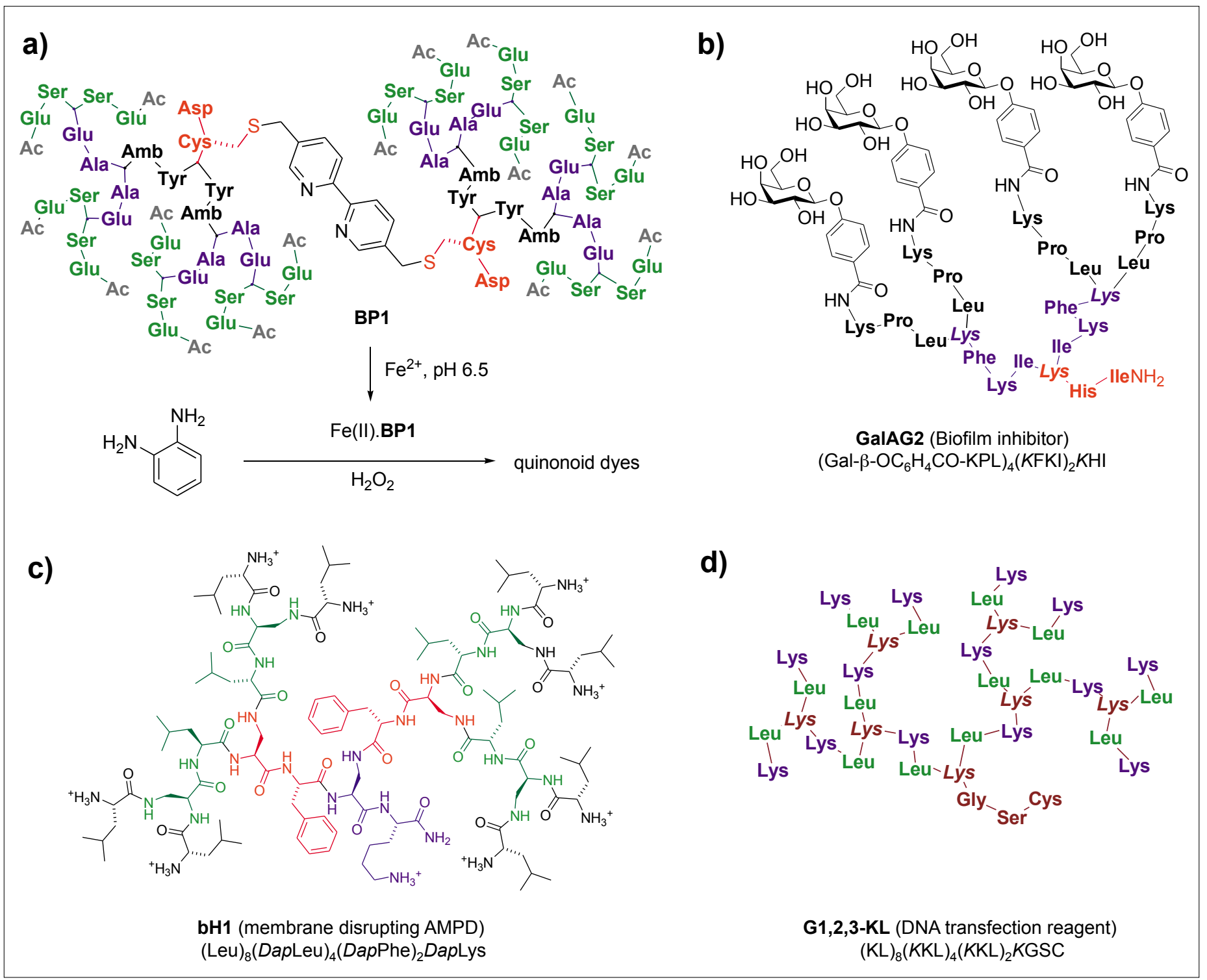

Fig. 2. a) Peptide dendrimers as peroxydase enzyme models, ${ }^{[22]}$ b) $P$. aeruginosa biofilm inhibitors, ${ }^{[25]}$ c) membrane disrupting AMPD, ${ }^{[29]}$ and d ) DNA transfection reagents. ${ }^{[32]}$ 
er branches to glutamines or by lowering the $\mathrm{pH}$ to 4.0 resulted in peptide dendrimers forming catalytically inactive $1: 3 \mathrm{Fe}$ dendrimer complexes.

\section{Antimicrobial Peptide Dendrimers}

Our more recent work on peptide dendrimers has focused on biological activities. ${ }^{[7]}$ As a first test case we investigated if peptide dendrimers could form a suitable scaffold to prepare glycoclusters by searching for multivalent inhibitors of the Pseudomonas aeruginosa lectin LecB, a fucose-specific lectin involved in biofilm formation in this human pathogen. An on-bead lectin binding assay identified several tight binding tetravalent fucosylated peptide dendrimers, ${ }^{[23]}$ among which peptide dendrimer FD2 turned out to be a particularly potent lectin ligand and biofilm inhibitor. ${ }^{[24]}$ The peptide dendrimer scaffold of FD2 was also used to prepare the tetravalent galactosylated peptide dendrimer and biofilm inhibitor GalAG2 by exchanging the terminal fucosides with galactosides groups (Fig. 2b). ${ }^{[25]}$ The glycopeptide dendrimer GalAG2 binds to the galactose specific $P$. aeruginosa lectin LecA via the galactosyl group and an unprecedented histidine $\mathrm{C}(\varepsilon) \mathrm{H}-\pi$ T-shape interaction between its aromatic aglycone and residue His50 on LecA. ${ }^{[26]}$ A systematic dendrimer mutagenesis study inspired by structure-based design has recently evidenced the importance of individual amino acid residues in lectin binding and biofilm inhibition by GalAG2, highlighting that the peptide dendrimer is not simply a tetravalent framework, but that its amino acid side-chains play an important role in biological activity. ${ }^{[27]}$

We have also investigated if peptide dendrimers might exhibit direct antimicrobial activities in analogy to the well-known antimicrobial peptides (AMP), which are often polycationic sequences with membrane-disrupting activities. ${ }^{[28]}$ To search for antimicrobial peptide dendrimers (AMPD), we adapted the above-mentioned 'off-bead' combinatorial library assay by spreading the photolysed SPPS beads on an agar plate inoculated with bacteria, and searched for clearing zones around active beads by staining live bacteria with the dye MTT after overnight incubation. [9] The method was first validated for the case of a focused library of analogues of the antimicrobial cyclic peptide natural product tyrocidin A, and subsequently applied to a peptide dendrimer combinatorial library.[29] The AMPD sequences identified in this combinatorial assay displayed hydrophobic residues at their surface and multiple positive charges in form of their amino termini, featuring an unprecedented type of antimicrobial agent. In particular dendrimer bH1 showed significant anti- microbial effects against various bacterial strains including $P$. aeruginosa (Fig. 2c). Mechanistic studies showed that the bactericidal effect involves membrane disruption, as evidenced by the interaction with phospholipid vesicles. In addition a molecular dynamics simulation was used to better understand how peptide dendrimer bH1 interacts with the bacterial membrane, and indicated that membrane penetration requires a substantial conformational rearrangement, an effect which is also observed by CD-spectroscopy. ${ }^{[30]}$ In ongoing experiments we have extended this combinatorial search and recently discovered AMPD against $P$. aeruginosa which also show potent activities against various multi-antibiotic resistant isolates.

\section{DNA Transfection Reagents and Cell-penetrating Peptide Dendrimers}

Peptide dendrimers can potentially serve as carriers for other bioactive agents or drugs to improve their efficacy. Several years ago we found that glycopeptide dendrimers could be used to deliver colchicine to cancer cells. ${ }^{[31]}$ More recently we have investigated the possibility of designing DNA transfection reagents on the basis of peptide dendrimers. The efficient delivery of DNA into cells is the prerequisite of the

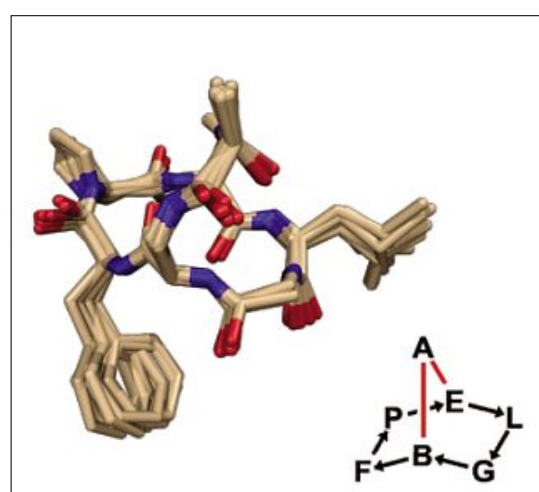

Norbornapeptide
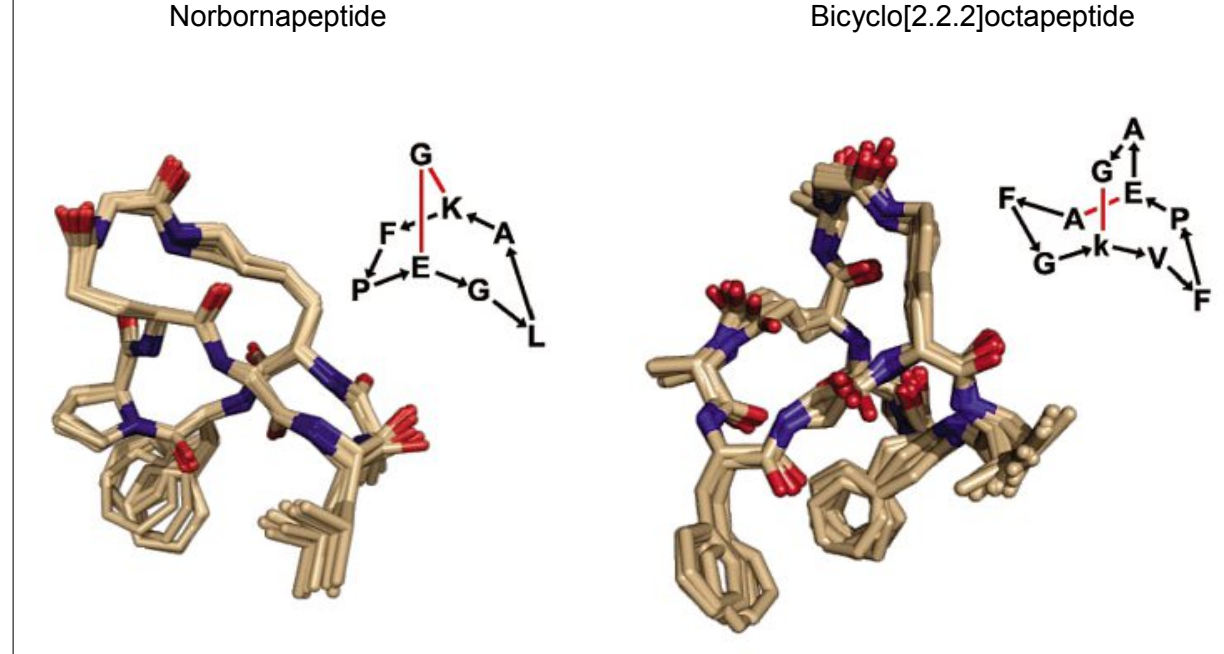

Fig. 3. Examples of bicyclic homodetic peptides and their structure as determined by ${ }^{1} \mathrm{H}$ NMR. ${ }^{111]}$

genetic manipulation of organisms in molecular and cellular biology and, ultimately, in nonviral gene therapy. By carrying out a thorough survey of G1, G2 and G3 peptide dendrimers with various combinations of cationic and hydrophobic residues for their gene delivery activity using a luciferase-based DNA transfection assay, we have identified peptide dendrimer G1,2,3-KL, which in co-application with a lipid component improves transfection by 6 to 10 -fold over commercial reagents under their respective optimal conditions (Fig. 2d).[32] The effect is quite striking and strongly depends on the amino acid sequence, whereby an even distribution of cationic and hydrophobic residues along the dendrimer branches is critical to obtain good DNA transfection.

Throughout our studies of bioactive peptide dendrimers the identification of cell-penetrating dendrimers has been a priority. To understand the effect of the dendritic topology on the cell-penetrating properties of peptides, we have carried out a systematic study on the cell-penetrating properties of $\mathrm{G} 1$ and $\mathrm{G} 2$ peptide dendrimers displaying two or four copies of known cell-penetrating peptides (CPP). [33] CPPs are peptides of 10 to 30 residues derived from natural translocating proteins. Multivalency was known to enhance cellu-

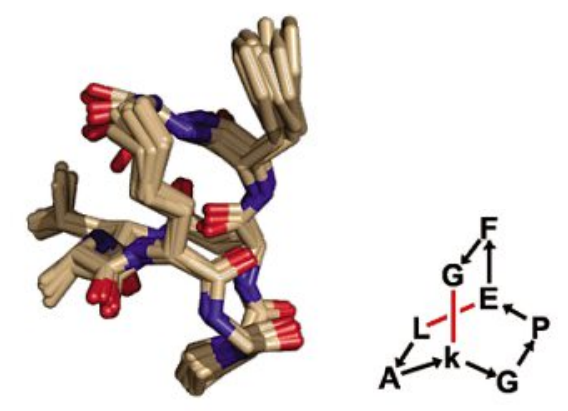

Bicyclo[2.2.2]octapeptide 
lar uptake for the Tat peptide and closely related polycationic sequences, however the generality of this effect on other sequences was unknown. A systematic comparison of dendrimers displaying the strongly cationic Tat, the amphipatic sequences Antp, pVEC and TP10, and the polyproline helix SAP for uptake in HeLa and CHO cells, intracellular localization, cytotoxicity and hemolysis was carried out. The study revealed that multivalency effects on uptake are indeed mostly found with polycationic sequences, but are strongly sequence dependent, providing guidelines for the future design of specific cell-penetrating peptide dendrimers.

\section{Polycyclic Peptides}

The use of branching amino acids as trivalent nodes in a peptide sequence makes it possible, in principle, to propose peptide analogues of a large diversity of polycyclic graphs. Surprisingly this approach to peptide design has not been considered systematically in the past, with only few reports on the synthesis of homodetic (in which amino acids are connected by amide bonds only) peptide analogues of polycyclic alkanes. ${ }^{[34]}$ We have initiated a program to investigate the synthesis and biological potential of polycyclic peptides by first establishing an SPPS protocol for simple bicyclic peptides comprising on-bead cyclisation, purification of the cyclic peptide, and closing of the second ring in solution. ${ }^{[11]}$ The approach was exemplified by preparing peptide topological equivalents of 'non-zero bridged' bicyclic alkanes such as norbornane. The structures were determined by ${ }^{1} \mathrm{H}$-NMR, which shows remarkable structural rigidity and slow exchange of backbone amide $\mathrm{NH}$ with the solvent (Fig. 3).

Bicyclic peptides define a very large structural family. For instance, there are 25 possible bicyclic peptide scaffolds of up to 10 amino acids forming $3.3 \cdot 10^{12}$ possible products using 20 different amino acids at the variable non-bridgehead positions. When considered from the point of view of molecular shape, it is interesting to note that bicyclic peptides populate a distinct area of peptide shape space. In particular, 'non-zero bridged' bicyclic peptides such as the norbornapeptide possess a significant third molecular dimension indicative of a globular shape, which should be particularly favourable for drug design including disruption of protein-protein interactions. Several ongoing projects in our group are investigating the potential of bicyclic and polycyclic peptides as scaffolds for selective binding to various targets.

\section{Conclusion and Outlook}

While SPPS is often perceived as a simplistic synthetic tool, its exploitation in the context of an expanded peptide topological space opens vast possibilities for molecular design. The potential of dendritic and polycyclic topologies for drug design is still largely unexploited. Branched peptides can be readily synthesized by SPPS capitalizing on well-established synthesis and purification methods as well as affordable reagents and scale-up possibilities. In the perspective of their possible use as drugs, we have found that both dendritic and polycyclic peptides are quite resistant to proteolysis and serum degradation. ${ }^{[35]}$ Furthermore the examples highlighted in this review provide ample evidence for their bioactivity potential. In each case we took advantage of combinatorial screening to rapidly identify active sequences and of SPPS to carry out detailed structure-activity relationship studies and optimize the activity by amino acid substitutions, insertions and deletions. This straightforward workflow enables the continued exploration of the peptide topological space in search for new drugs.

\section{Acknowledgement}

This work was supported financially by the University of Bern, the Swiss National Science Foundation, the COST Actions D34 and CM1201, and the Marie-Curie ITN BioChemLig.

Received: October 9, 2013

[1] B. Merrifield, Methods Enzymol. 1997, 289, 3.

[2] C. L. Stevenson, Curr. Pharm. Biotechnol. 2009, 10, 122.

[3] L. Cascales, D. J. Craik, Org. Biomol. Chem. 2010, 8,5035 .

[4] C. Chatterjee, M. Paul, L. Xie, W. A. van der Donk, Chem. Rev. 2005, 105, 633.

[5] T. Karskela, P. Virta, H. Lonnberg, Curr. Org. Synth. 2006, 3, 283.

[6] a) J.-L. Reymond, L. C. Blum, R. van Deursen, Chimia 2011, 65, 863; b) L. Ruddigkeit, R. Van Deursen, L. C. Blum, J.-L. Reymond, J. Chem. Inf. Model. 2012, 52, 2864.

[7] J.-L. Reymond, T. Darbre, Org. Biomol. Chem 2012, 10, 1483.

[8] J. Kofoed, J.-L. Reymond, J. Comb. Chem. 2007, 9, 1046

[9] V. S. Fluxa, N. Maillard, M. G. P. Page, J.-L. Reymond, Chem. Commun. 2011, 47, 1434

[10] N. Maillard, R. Biswas, T. Darbre, J.-L. Reymond, ACS Comb. Sci. 2011, 13, 310.

[11] M. Bartoloni, R. U. Kadam, J. Schwartz, J. Furrer, T. Darbre, J.-L. Reymond, Chem. Commun. 2011, 47, 12634

[12] A. Esposito, E. Delort, D. Lagnoux, F. Djojo, J.-L. Reymond, Angew. Chem., Int. Ed. 2003 , 42, 1381.

[13] J.-L. Reymond, J. Immunol. Methods 2002 $269,125$.

[14] J.-L. Reymond, V. S. Fluxa, N. Maillard, Chem. Commun. 2009, 34.
[15] a) A. Clouet, T. Darbre, J.-L. Reymond, Angew. Chem., Int. Ed. 2004, 43, 4612; b) J. Kofoed, J.-L. Reymond, Chem. Commun. 2007, 4453.

[16] N. Maillard, T. Darbre, J.-L. Reymond, J. Comb. Chem. 2009, 11, 667.

[17] a) E. Delort, N. Q. Nguyen-Trung, T. Darbre, J.-L. Reymond, J. Org. Chem. 2006, 71, 4468; b) J. Kofoed, T. Darbre, J.-L. Reymond, Org. Biomol. Chem. 2006, 4, 3268; c) R. Biswas, N. Maillard, J. Kofoed, J.-L. Reymond, Chem. Commun. 2010, 46, 8746.

[18] P. Sommer, N. A. Uhlich, J.-L. Reymond, T. Darbre, ChemBiochem 2008, 9, 689.

[19] N. A. Uhlich, A. Natalello, R. U. Kadam, S. M. Doglia, J.-L. Reymond, T. Darbre, ChemBiochem 2010, 11, 358 .

[20] N. A. Uhlich, P. Sommer, C. Buhr, S. Schurch, J.-L. Reymond, T. Darbre, Chem. Commun. 2009, 6237.

[21] N. A. Uhlich, T. Darbre, J.-L. Reymond, Org. Biomol. Chem. 2011, 9, 7071 .

[22] P. Geotti-Bianchini, T. Darbre, J.-L. Reymond, Org. Biomol. Chem. 2013, 11, 344.

[23] E. Kolomiets, E. M. Johansson, O. Renaudet, T. Darbre, J.-L. Reymond, Org. Lett. 2007, 9, 1465.

[24] E. M. Johansson, S. A. Crusz, E. Kolomiets, L. Buts, R. U. Kadam, M. Cacciarini, K. M. Bartels, S. P. Diggle, M. Camara, P. Williams, R. Loris, C. Nativi, F. Rosenau, K. E. Jaeger, T. Darbre, J.-L. Reymond, Chem. Biol. 2008, 15, 1249.

[25] R. U. Kadam, M. Bergmann, M. Hurley, D. Garg, M. Cacciarini, M. A. Swiderska, C. Nativi, M. Sattler, A. R. Smyth, P. Williams, M. Camara, A. Stocker, T. Darbre, J.-L. Reymond, Angew. Chem., Int. Ed. 2011, 50, 10631.

[26] R. U. Kadam, D. Garg, J. Schwartz, R. Visini, M. Sattler, A. Stocker, T. Darbre, J.-L. Reymond, ACS Chem. Biol. 2013, 8, 1925.

[27] a) J.-L. Reymond, M. Bergmann, T. Darbre, Chem. Soc. Rev. 2013, 42, 4814; b) R. U. Kadam, M. Bergmann, D. Garg, G. Gabrieli, A. Stocker, T. Darbre, J.-L. Reymond, Chem. Eur, J. 2013, doi: 10.1002/chem. 201302587.

[28] S. P. Liu, L. Zhou, R. Lakshminarayanan, R. W. Beuerman, Int. J. Pept. Res. Ther. 2011, 16, 199.

[29] M. Stach, N. Maillard, R. U. Kadam, D. Kalbermatter, M. Meury, M. G. P. Page, D. Fotiadis, T. Darbre, J.-L. Reymond, Medchemcomm 2012, 3, 86.

[30] H. K. Ravi, M. Stach, T. A. Soares, T. Darbre, J.-L. Reymond, M. Cascella, Chem. Commun. 2013, 49,8821

[31] E. M. Johansson, J. Dubois, T. Darbre, J.-L. Reymond, Bioorg. Med. Chem. 2010, 18, 6589.

[32] A. Kwok, G. A. Eggimann, J.-L. Reymond, T. Darbre, F. Hollfelder, ACS Nano 2013, 7, 4668.

[33] G. A. Eggimann, S. Buschor, T. Darbre, J.-L. Reymond, Org. Biomol. Chem. 2013, 11, 6717.

[34] a) M. Teixido, M. Altamura, L. Quartara, A. Giolitti, C. A. Maggi, E. Giralt, F. Albericio, J. Comb. Chem. 2003, 5, 760; b) R. Hirschmann, W. Q. Yao, B. Arison, L. Maechler, P. A. Sprengeler, A. B. Smith, Tetrahedron Lett. 1996, 37, 5637; c) J. C. Tolle, M. A. Staples, E. R. Blout, J. Am. Chem. Soc. 1982, 104, 6883; d) C. Bracken, J. Gulyas, J. W. Taylor, J. Baum, J. Am. Chem. Soc. 1994, 116, 6431; e) R. Oliva, L. Falcigno, G. D’Auria, M. Saviano, L. Paolillo, G. Ansanelli, G. Zanotti, Biopolymers 2000, 53, 581.

[35] a) P. Sommer, V. S. Fluxa, T. Darbre, J.-L. Reymond, ChemBiochem 2009, 10, 1527; b) E. M. V. Johansson, R. U. Kadam, G. Rispoli, S. A. Crusz, K.-M. Bartels, S. P. Diggle, M. Camara, P. Williams, K.-E. Jaeger, T. Darbre, J.-L. Reymond, Medchemcomm 2011, 2, 418. 